\title{
A Model of Convergent Extension in Animal Morphogenesis
}

\author{
Mark Zajac*, Gerald L. Jones and James A. Glazier \\ University of Notre Dame, Department of Physics Notre Dame, IN 46556
}

(October 24, 2018)

\begin{abstract}
In this paper we argue that the pattern of cell movements in the morphogenetic process known as convergent extension can be understood as an energy minimization process, provided the cell-cell adhesive energy has a certain type of anisotropy. This single simple property suffices to cause the cell elongation, alignment, and intercalation of a cellular array that are the characteristics of convergent extension. We describe the type of anisotropy required. We show that the final aspect ratio of the array of cells is independent of the initial configuration and of the degree of cell elongation. We find how it depends on the anisotropy.
\end{abstract}

*mzajac@krypton.helios.nd.edu 

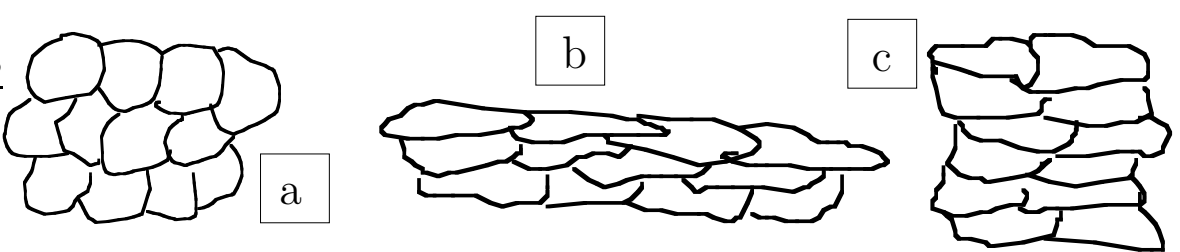

FIG. 1. Intercalation. Isodiametric cells (a) become elongated and aligned (b)while simultaneously intercalating (c) so that an array of cells extends at right angles to the direction of cell motion.

In the development of the animal embryo great changes of form (morphogenesis) take place [自], especially during gastrulation when axial structures are formed by extensive cell rearrangement. During these rearrangements groups of cells move coherently over distances very large compared to cell dimensions. This process has been extensively investigated by experiments, particularly on embryos of the frog, Xenopus laevis, and particularly by R.E. Keller and his collaborators (see [2] for a brief review and extensive references).

One characteristic and widespread type of rearrangement, "convergent extension", occurs, for example, in the development of axial structure such as precursors to the vertebrate spinal column. Here an active group of cells undergoes a threefold process. The individual cells, originally roughly isodiametric (Fig. 1 1a), elongate and their axes of elongation become aligned. If these were the only motions the final configuration would be as in Fig. 11b. But at the same time, though on a somewhat slower time scale, the cells intercalate between each other. The intercalation is in the direction of alignment so that the number of cells in that direction decreases while the number of cells in directions perpendicular to the alignment increases producing a final configuration as in Fig. [lc. The elongation increases the overall length of the group of cells in the direction of alignment and decreases the length in orthogonal directions (since the volume stays roughly constant). Intercalation does the reverse but dominates, so that the axis of net extension of the group of cells is at right angles to the axis of individual cell elongation. Here we argue that the important aspects of convergent extension will result from a tendency of the active cells to minimize their total energy, provided that they interact with a non-uniform surface (adhesive) energy satisfying certain conditions. We also develop a mean field theory of this process.

Minimum energy principles have been used to explain cell rearrangement since Steinberg's [3] suggestion that differential cell adhesion plus cell motility can account for cell sorting patterns in mixtures of two or more cell types (see [4] for a review and extensive references to the literature). Goel and Lieth [5] have considered cell sorting for a simple geometrical model in the presence of anisotropic surface adhesion between cells of fixed shape. Cell sorting, driven by energy minimization, has also been the subject of many computer simulations [6.7]. Drasdo, Kree, and McCaskill [8] have simulated cell sorting with anisotropic surface adhesion. Anisotropic surface adhesion has not, so far, been used to explain the convergent extension of a homogeneous group of cells. We do not model here the dynamics of convergent extension. We assume, as in [3] and [5], that cell motility will allow the system to explore its possible configurations and that, as a strongly dissipative system, it will evolve towards the configuration of minimum energy.

In the embryo convergent extension usually takes place in an asymmetric environment where the inactive cells bounding the active region are not the same on all sides of that 

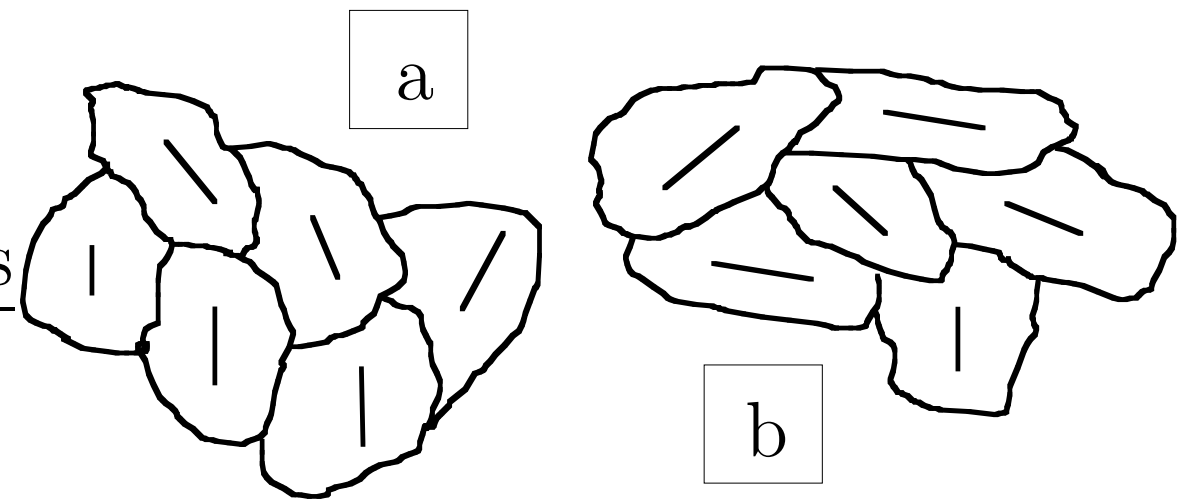

FIG. 2. Cell alignment. For an ordered array (a) most cell attachments are either end to end or side to side while a disordered array (b) exhibits significant binding between poles and lateral surfaces.

region. In this case, the extension, and its orientation, may be determined by the interactions at the boundaries which "channel" the active cells, rather than being an intrinsic collective property of the group of active cells. Under these experimental circumstances the boundaries strongly influence active cell movements. Indeed, in the physical model of Weliky et.al. [9], convergent extension results only if active cells at the boundaries parallel to the elongation, behave differently from those at the boundaries perpendicular to the elongation.

A subsequent and elegant experiment by Shih and Keller [10], however, strongly suggests that, in addition, the active cells have a strong intrinsic collective mechanism driving their convergent extension. In these experiments a layer (essentially a monolayer) of active cells was excised from a frog embryo, at a stage before convergent extension had begun, and cultured on a uniform surface in a medium which allowed their normal development. Subsequently the layer showed strong convergent extension in the plane of the substrate and this in the absence any plausible lateral anisotropy either in the substrate or in the culture medium. This behavior thus appears to be an example of "broken symmetry" so well known in condensed matter physics, and asks for an explanation based on collective behavior induced by cell-cell interactions.

To explain this behavior by energy minimization we assume that cell-cell interactions take place through surface adhesion, which can be characterized by an energy per unit contact area. We assume that the cell rearrangements take place with negligible cell division and little change in cell volume, as is observed in the later stages of the above experiment. There is no clear understanding in the literature of the trigger for the cell elongation which initiates the convergent extension, and our model does not provide this. Our main assumption is that the adhesive energy of the contact surface between two cells will depend on how that surface is oriented relative to the axes of elongation of the two cells. This would be so, for example, were the surface density of adhesive binding sites to be different on the long side of a cell (parallel to the axis of elongation) from that on the short sides (perpendicular to the axis of elongation). We can find in the literature no compelling evidence either for or against this assumption. We argue here that a specific form of this assumption is a sufficient cause of the elongation, alignment, and intercalation resulting in convergent extension.

We give here a two-dimensional version of our proposal since the convergent extension takes place in the plane of the substrate and the height of the cells does not seem to play an 
important role. Hence we consider a collection of two dimensional cells of (nearly) the same fixed area. We first want to find the conditions which favor alignment. We assume a compact array of cells large enough that array surface effects are (for this argument) negligible. Fig. $2 \mathrm{a}$ is a cartoon of a few elongated cells in such a large ordered array of cells and Fig. 20 b is for a disordered array. Suppose that we can roughly distinguish, for each cell, two long sides (parallel to the axis of elongation) and two short sides (perpendicular). Figure la shows that in the ordered array the cell-cell contact surfaces are, for the most part, either roughly parallel to the common axes of alignment or roughly perpendicular to that axis. We shall term these long-long $(l l)$ or short-short $(s s)$ contacts since they occur, primarily, at contacts between a pair of long sides or a pair of short sides. The disordered array of Fig. 2 $2 \mathrm{~b}$ has many contact surfaces that make intermediate angles with the now different axes of adjacent cells. We term these long-short $(l s)$ contacts since they tend to occur whith the contact surfaces between a long side of one cell and a short side of a neighbor. If the energy density (per unit length) of the $l s$ contacts is enough larger than those of $l l$ and $s s$ contacts then the ordered array will have the lower energy per cell (we have assumed that the array is large enough that we can neglect the effect of the array boundaries on the bulk ordering). More quantitatively, let $l$ and $s$ be the average long and short side lengths of each cell, which, for the moment, we take as fixed. Suppose that all cell-cell contacts can be characterized as $l l, s s$, or $l s$ and that the total lengths of each type in the array are $L_{l l}, L_{s s}$, and $L_{l s}$. In a large array of $N$ cells we have $2 N l=2 L_{l l}+L_{l s}$ and $2 N s=2 L_{s s}+L_{l s}$ (again neglecting array boundaries where cells do not contact other cells). Since $N, l$ and $s$ are fixed these equations provide two constraints between the three contact lengths. We assume that three energy densities $\left(J_{l l}, J_{s s}\right.$, and $\left.J_{l s}\right)$ are adequate to characterize the interactions at the various surfaces. Then the bulk energy of an array due to cell-cell interactions is

$$
\begin{aligned}
E & =L_{l l} J_{l l}+L_{s s} J_{s s}+L_{l s} J_{l s} \\
& =\left(2 N l-L_{l s}\right) J_{l l} / 2+\left(2 N s-L_{l s}\right) J_{s s} / 2+L_{l s} J_{l s} \\
& =\left(J_{l s}-J_{l l} / 2-J_{s s} / 2\right) L_{l s}+N\left(l J_{l l}+s J_{s s}\right) .
\end{aligned}
$$

This energy is an increasing function of $L_{l s}$ if the ordering condition:

$$
\gamma_{l s}=J_{l s}-\left(J_{l l}+J_{s s}\right) / 2>0,
$$

is satisfied. In this event ordered arrays $\left(L_{l s}=0\right)$ will have lower bulk energies than disordered $\left(L_{l s}>0\right)$ arrays. Note that condition (2) is just that the $l s$ surface tension $\gamma_{l s}$ be positive.

The above argument is exact if the cells are assumed (unrealistically) to be identical rectangles arranged in arbitrary tesselations of the plane and is similar to that used in [5] in the cell sorting problem. For realistic cells it is a crude but plausible representation of the assumed anisotropy of the surface adhesion.

The case where the adhesive energy density of a two cell contact is the product of a factor from each cell is interesting. For example, where a variation in the density of the binding sites on the cell surface causes the variation in energy. If we make the natural assumption that the density of adhesive bonds is proportional to the product of the density of binding sites on the cell surfaces in contact, then we would have in the above model $J_{l l}=-j_{l} j_{l}$, 
$J_{s s}=-j_{s} j_{s}$, and $J_{l s}=-j_{l} j_{s}$, where the sign is chosen make all $J<0$ when all $j>0$. This choice satisfies the ordering condition Eq. (1) whenever $j_{l}$ and $j_{s}$ are positive and are not equal.

In addition to Eq. (1) let us suppose that the $l l$ energy density is lower than the $s s$ energy density.

$$
J_{l l}<J_{s s}<0\left(\text { or } j_{l}>j_{s}>0\right) .
$$

Now the energy Eq. (11) of the array can be reduced by increasing the cell long side length $l$ and decreasing the short side length $s$ causing, or at least favoring, elongation of the cells. At equilibrium these surface effects will presumably be balanced by internal cellular forces opposing further elongation.

We can also argue that Eq. (3) will produce intercalation in the direction of elongation. Consider the effect of the boundary on a finite array of $\mathrm{N}$ cells. Suppose that boundary cells have no adhesive energy with the culture medium. Then the expression Eq. (1) underestimates the array energy because it assumes all cell surfaces are in contact with other cell surfaces and so overestimates the contact lengths $L_{l l}$ and $L_{s s}$. From Eq. (1) we should subtract the (negative) adhesive energy that is not present at the contacts between the boundary cells and the surrounding medium. Figure 1 shows arrays of twelve elongated cells. In Fig. 1]c the array extension is at right angles to the cell elongation and in Fig. [1b it is along the cell elongation. Clearly in Fig. 1/c the boundary contacts are primarily through short cell sides whereas in Fig. 1]b they are primarily though long cell sides. Since the long sides have lower (more negative) energy than the short, the energy (corrected for boundaries) of the configuration shown in Fig. [b is higher than that of Fig. 11c. Thus if we start with any compact initial array of unelongated cells we expect cell motility and energy minimization to produce configuration of type Fig. 1]c by cell elongation, alignment, and intercalation parallel to the alignment. In order for these processes to produce net extension in the direction perpendicular to alignment the effects of intercalation must dominate those of elongation. For a rectangular array of a large number of rectangular cells a direct calculation shows that this will be so, independent of the degree of elongation, and that the ratio of the array dimensions in the directions perpendicular and parallel to the elongation is $J_{l l} / J_{s s}$. We derive these results more generally below.

We can make the above arguments concerning surface effects somewhat more realistic and quantitative by the following mean field type of modeling. We assume that we have a large array of $N$ elongated and aligned cells. The total energy of the array is the bulk energy due to cell-cell interactions plus the surface correction for the absence of cells outside the boundary. The bulk energy is proportional to $N$, or equivalently, the array area $A$, so we write it as $\lambda A$, where $\lambda$ is the (negative) bulk energy per unit area in the aligned array. To model the anisotropic cell-cell interaction we assume that $J$ depends on the angle between the direction of alignment, specified by the unit vector $\hat{\mathbf{a}}$, and the unit vector $\hat{\mathbf{n}}$ normal to the contact segment between the cells (see Fig. Ba). More explicitly, we assume that $J(\hat{\mathbf{n}} \cdot \hat{\mathbf{a}})$ is negative, an even function (since $\hat{\mathbf{a}},-\hat{\mathbf{a}}$ and $\hat{\mathbf{n}},-\hat{\mathbf{n}}$ specify the same physical situations), and is minimum at $\hat{\mathbf{n}} \cdot \hat{\mathbf{a}}=0$ (so that $l l$ interactions have the lowest energy). Figure $3 \mathrm{~b}$ shows part of a finite array of vertically aligned cells and their boundary with an external medium with which we assume they have no adhesive energy. To get the energy of the finite array 

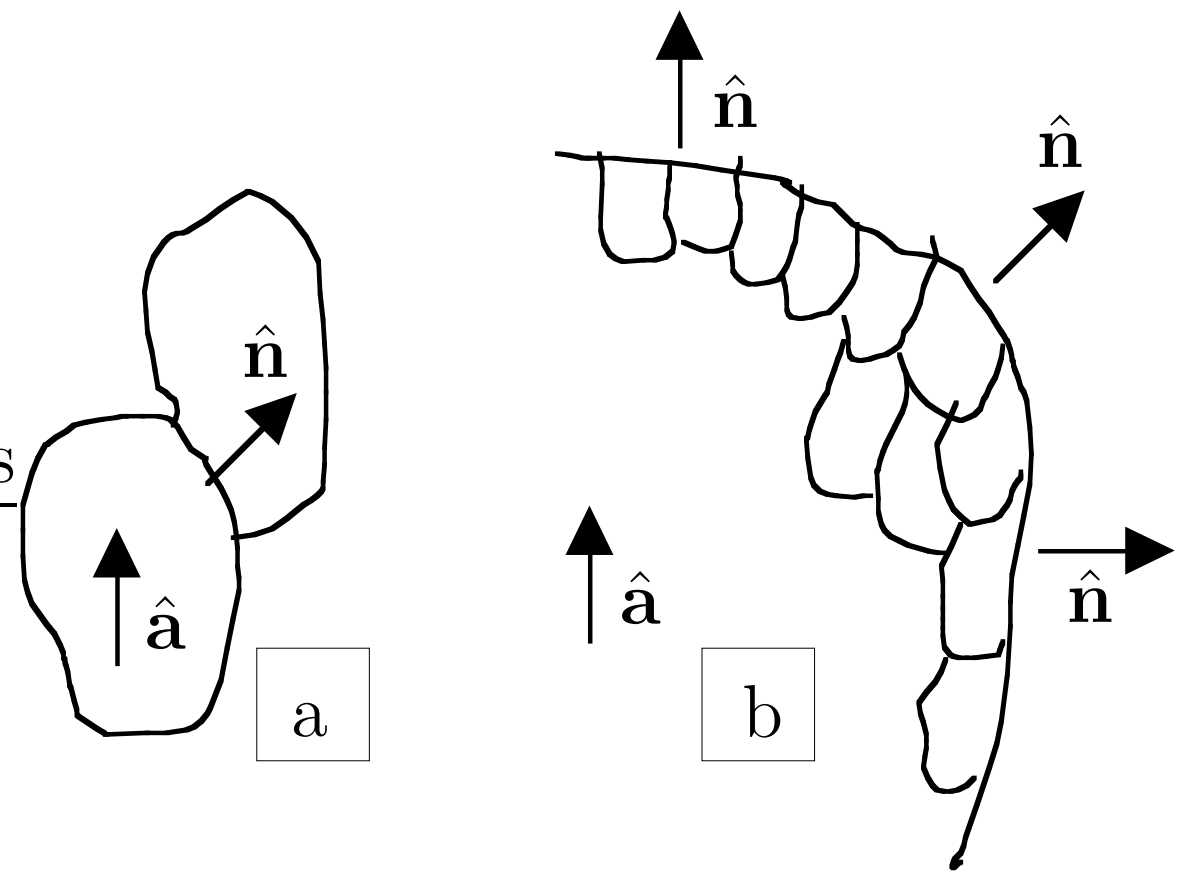

FIG. 3. Anisotropic binding. Adhesive energy at the point of contact between cells (a) is assumed to depend on $(\hat{\mathbf{n}} \cdot \hat{\mathbf{a}})^{2}$ where $\hat{\mathbf{n}}$ is the local unit normal while $\hat{\mathbf{a}}$ gives alignment, assumed common to all cells. At an interface with uniformly inert surroundings (b), missing adhesive energy will vary with the orientation of the surface cells, relative to the boundary.

we must subtract from the bulk energy half the energy the boundary cells would have had with cells external to the array had the boundary been absent. Half, since adhesive energy is shared between two cells. So:

$$
E=\lambda A-\frac{1}{2} \oint J(\hat{\mathbf{n}} \cdot \hat{\mathbf{a}}) \mathrm{d} l,
$$

where the integral is taken around a closed boundary. We want to minimize this over all closed boundaries enclosing the same area $A$. Alternatively we can interpret $\lambda$ as a Lagrange multiplier and find the extrema of Eq. (4) over all closed curves at fixed $\lambda$. To do this parameterize the curves as $\mathbf{r}(u)$ with $0 \leq u \leq 1$, and $\mathbf{r}(0)=\mathbf{r}(1)$. Then, since $\mathrm{d} l=$ $\left(\dot{x}^{2}+\dot{y}^{2}\right)^{1 / 2} \mathrm{~d} u($ where $\dot{\mathbf{r}}=d \mathbf{r} / d u)$, while $(\hat{\mathbf{n}} \cdot \hat{\mathbf{a}})=\left(a_{y} \dot{x}-a_{x} \dot{y}\right) /\left(\dot{x}^{2}+\dot{y}^{2}\right)^{1 / 2}$ and $A=\int_{0}^{1} y \dot{x} \mathrm{~d} u$ we can write the energy as $\int_{0}^{1} \mathcal{L}(\mathbf{r}, \dot{\mathbf{r}}) \mathrm{d} u$ with $\mathcal{L}(\mathbf{r}, \dot{\mathbf{r}})=\lambda y \dot{x}-J(\hat{\mathbf{n}} \cdot \hat{\mathbf{a}})\left(\dot{x}^{2}+\dot{y}^{2}\right)^{1 / 2} / 2$. The extremal curves are solutions of the usual Euler-Lagrange equations for $\mathcal{L}$ and are degenerate with respect to translations in the $x-y$ plane. This degeneracy gives rise to two first integrals and two constants of integration (which we choose to be zero), which fix the position of the extremal curve. The integrated equations have the form

$$
2 \lambda \mathbf{r}=\hat{\mathbf{a}} J^{\prime}(\hat{\mathbf{n}} \cdot \hat{\mathbf{a}})+\hat{\mathbf{n}}\left[J(\hat{\mathbf{n}} \cdot \hat{\mathbf{a}})-(\hat{\mathbf{n}} \cdot \hat{\mathbf{a}}) J^{\prime}(\hat{\mathbf{n}} \cdot \hat{\mathbf{a}})\right],
$$

where $J^{\prime}$ is the derivative of $J$. The dot product of Eq. 5 with $\hat{\mathbf{r}}$ gives the Wulff condition [11]. Equation (5) is two coupled first order differential equations whose solutions depend on the particular choice of the function $J$. We have not been able to find complete analytic solutions for any interesting choice of $J$ but some properties of the solutions can be found. 
First we note that for $\hat{\mathbf{a}}=0$, or equivalently $J=$ constant, the solution is a circle of radius $J /(2 \lambda)$. Secondly, the turning points of any solution curve are where $d(\mathbf{r} \cdot \mathbf{r}) / d u=0$. Now:

$$
\lambda \frac{d}{d u}(\mathbf{r} \cdot \mathbf{r})=2 \lambda(\dot{\mathbf{r}} \cdot \mathbf{r})=(\dot{\mathbf{r}} \cdot \hat{\mathbf{a}}) J^{\prime}(\hat{\mathbf{n}} \cdot \hat{\mathbf{a}})
$$

where we have used Eq. (5) and that $\dot{\mathbf{r}} \cdot \hat{\mathbf{n}}=0$ for any curve. From Eq. (6) we find two types of turning points. 1) At $\dot{\mathbf{r}} \cdot \hat{\mathbf{a}}=0$, that is, where the boundary is perpendicular to the alignment so that $\hat{\mathbf{n}}= \pm \hat{\mathbf{a}}$. For any simple closed curve this condition will be satisfied at two points on the curve. Because $J$ is even and $J^{\prime}$ is odd we have from (5) that these two points lie at $\pm J(1) /(2 \lambda)$ on the line through the origin and parallel to $\hat{\mathbf{a}}$. 2) At $\hat{\mathbf{n}} \cdot \hat{\mathbf{a}}=0$ where $J^{\prime}=0$ and the boundary is parallel to the alignment. For these (5) shows that $2 \lambda \mathbf{r}=\hat{\mathbf{n}} J(0)$, thus there are turning points at $\pm J(0) /(2 \lambda)$ along a line through the origin and perpendicular to $\hat{\mathbf{a}}$. If we let $D_{\perp}$ and $D_{\|}$be the distances between the turning points aligned respectively perpendicular and parallel to $\hat{\mathbf{a}}$ then the aspect ratio of the boundary is

$$
D_{\perp} / D_{\|}=J(0) / J(1)
$$

If $J(1)<J(0)<0$ then $D_{\perp} / D_{\|}>1$ and the elongation is in the direction perpendicular to the alignment, as is observed in convergent extension. From Fig. 3 a we see that $J(0)$ corresponds to our previous $J_{l l}$ while $J(1)$ corresponds to $J_{s s}$.

We have also studied the minimization of the energy functional Eq. (4) numerically for the case where $J$ is chosen to be a gaussian function. We approximate the boundary curve by a polygon of at least 100 sides and use an iterative process that moves down the energy gradient at constant area. We have started from many initial configurations, all of which are simple closed polygons. The final boundary curve is always the same and with the correct aspect ratio Eq. (7). This iterative process could also be viewed as a model for the dynamics of convergent extension. Indeed, with the addition of additive random forces, the method would be essentially a Langevin dynamics for the evolution of a highly dissipative system.

In conclusion, we can understand convergent extension as an energy minimization process, provided the cell-cell adhesive energy has a certain kind of anisotropy. This single simple property is sufficient cause for the cell extension, alignment, and intercalation in the direction of alignment, that are the characteristics of convergent extension. We have characterized the anisotropy required [Eq. (2) and Eq. (3)]. We have shown that the final aspect ratio is independent of the initial configuration and have shown how it depends on the anisotropy Eq. (7).

We believe our arguments are plausible but realize that they are not conclusive. Our modeling neglects many degrees of freedom associated with cell shape and arrangement, which we think, but cannot prove, are not crucial. Our procedure of separately minimizing the bulk and surface energies is accurate only for a large array of cells. We do not see much possibility of doing a lot better by purely analytic methods. We have initiated simulations of convergent extension, using the Potts model and Metropolis dynamics methods of references [6] and [7], with anisotropic adhesive energies of the type described in this paper. The use of anisotropic adhesive energies introduces technical difficulties in that the energy becomes non-local on the scale of the size of a cell, which considerably increases the simulation time. Nevertheless we believe the simulations will eventually substantiate our conclusions. Even 
so, the more difficult question of whether this explanation of convergent extension is correct remains. Experiments that probe the possible anisotropy of cell adhesive energy would be

useful, as would experiments that show the final configuration is largely independent of the initial configuration.

\section{ACKNOWLEDGMENTS}

The authors thank Francois Graner for a helpful discussion. 


\section{REFERENCES}

[1] L. Wolpert et al., Principles of Development (Oxford University Press, New York, 1998).

[2] R. Keller and J. Shih, in Interplay of Genetic and Physical Processes in the Development of Biological Form at the Frontier of Physics and Biology, Les Houches, edited by D. Beysens, G. Forgacs, and F. Gail (World Scientific, Singapore, 1995), pp. 143-153.

[3] M. S. Steinberg, Science 141, 401 (1963).

[4] F. Graner, J. theor. Biol. 164, 455 (1993).

[5] N. S. Goel and A. G. Leith, J. theor. Biol. 28, 469 (1970).

[6] F. Graner and J. A. Glazier, Phys. Rev. Lett. 69, 2013 (1992).

[7] J. A. Glazier and F. Graner, Phys. Rev. E 47, 2128 (1993).

[8] D. Drasdo, R. Kree, and J. S. McCaskill, Phys. Rev. E 52, 6635 (1995).

[9] M. Weliky, S. Minsuk, R. Keller, and G. Oster, Development 113, 1231 (1991).

[10] J. Shih and R. Keller, Development 116, 887 (1992).

[11] G. Wulff and Z. Kristall, Mineral 34, 449 (1901); I. V. Markov, Crystal Growth for Beginners (World Scientific, Singapore, 1995). 